\title{
A Task Allocation Model for a Team of Aircraft Launching on the Carrier
}

\author{
Jiapeng Cui, ${ }^{1}$ Yu Wu $\mathbb{D}^{1},{ }^{1}$ Xichao Su, ${ }^{2}$ and Jingyu Song ${ }^{3}$ \\ ${ }^{1}$ College of Aerospace Engineering, Chongqing University, Chongqing 400044, China \\ ${ }^{2}$ Naval Aviation University, Yantai 264001, China \\ ${ }^{3}$ System Engineering Research Institute, China State Shipbuilding Corporation, Beijing 10094, China \\ Correspondence should be addressed to Yu Wu; cquwuyu@cqu.edu.cn
}

Received 3 January 2018; Accepted 11 April 2018; Published 20 May 2018

Academic Editor: Thomas Hanne

Copyright (C) 2018 Jiapeng Cui et al. This is an open access article distributed under the Creative Commons Attribution License, which permits unrestricted use, distribution, and reproduction in any medium, provided the original work is properly cited.

High level efficiency and safety are of paramount importance for the improvement of fighting capability of an aircraft carrier. The task allocation problem for a team of aircraft launching on the carrier is studied in this paper. Although the study of this problem is of great significance, no relevant literature has been found on this issue. Firstly, the conceptual model of problem is formulated with the planning objectives and the constraints defined. Then the multi-aircraft and multi-catapult launching task allocation problem is decomposed into two consecutive sub-tasks, that is, catapult allocation and the launching sequence determination. The taxi time of aircraft is considered during the catapult allocation process, and the launching position of each aircraft is determined using a decision-making method. In the launching sequence determination step, the starting collision risk of aircraft is introduced to optimize the launching sequence which results in the minimum collision risk on each catapult. Thirdly, the proposed method is validated using the setups of the Nimitz-class aircraft carrier. The proposed method is used to solve the task allocation problem and is compared to the artificial heuristics approach and the brute force approach. Experiment results demonstrate that the proposed method has better performance than the artificial heuristics approach and has better performance than the brute force approach in balancing efficiency and safety.

\section{Introduction}

Aircraft carrier plays an essential role in defending the security of territorial waters and safeguarding maritime interests [1]. There are various tasks, such as launching, taxiing and landing, operated on the flight deck of carrier, and it is important to arrange these tasks in the limited space [2]. Otherwise, the normal operation of the aircraft carrier system cannot be assured $[3,4]$. The efficiency and safety of launching mission play an essential role in dominating the fighting capability of the aircraft carrier [5], and a reasonable automatic task allocation approach is imperative for a team of aircraft launching on the carrier.

The study of task allocation develops with the modern information technology. It has been extensively noted in the planning system of aircraft, satellites, ground vehicle, and robot $[6,7]$. Vehicle is scheduled with some tasks at a time with the existing resources $[8,9]$, and the ultimate goal is to optimize the proposed performance index under various constraints [10]. As the number of aircraft increases, the flight mission becomes complicated. Multiple aircraft are required to complete the mission cooperatively under the dynamic environment because it increases the probability of a successful mission $[11,12]$. To make the complicated mission simple, the mission is usually decomposed into a series of basic tasks so that the tasks can be executed directly by every aircraft [13-15]. Hierarchical planning method is effective in dealing with the complicated task allocation problem $[16,17]$. The method provides a platform for optimizing the outcomes, and the established mathematical models could apply in a variety of more complex mission scenarios.

The aircraft departure scheduling problem (ADSP) is similar to the task allocation of aircraft launching on the carrier (TAALC) and has been widely studied. The two problems both seek to determine the sequence of aircraft taking off from the available runways/catapults on airports/carrier 


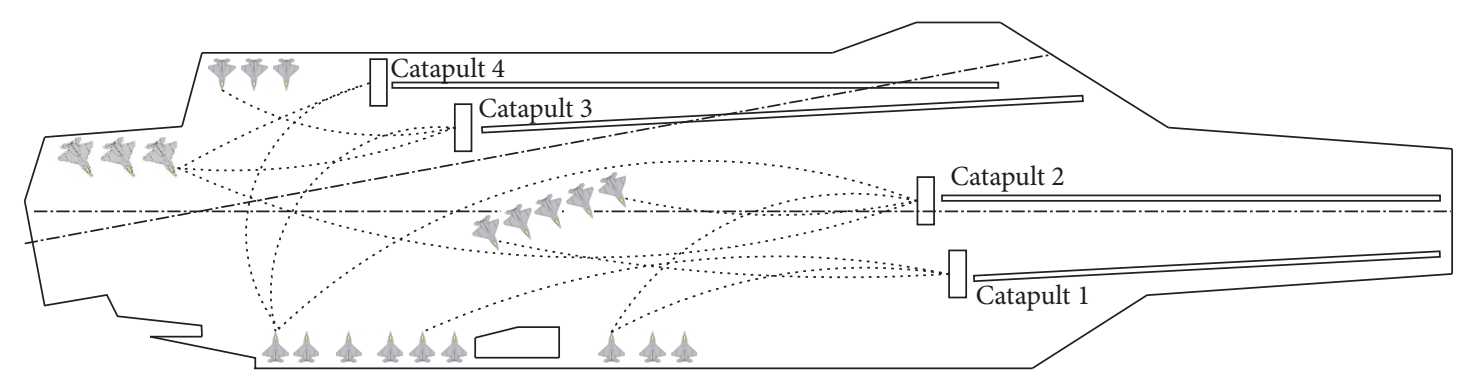

Parking aircraft

FIGURE 1: General environment of flight deck and the description of task allocation problem.

in order to optimize given objectives subject to a variety of operational constraints. Dynamic programming [18], branch and bound [19], and heuristics and meta-heuristics [20] are the main solution techniques. In general, ADSP and TAALC are both treated as examples of the generic job-shop scheduling problem.

Compared to ASDP, stricter constraints are imposed on TAALC. To be specific, the narrow space of flight deck results in more risks. As the launching and landing of aircraft are proceed on the flight deck in a cycling way, any delay may disturb the tempos under such a time-critical scheduling. Moreover, the constraints are more complicated in the flight deck operation of carrier.

Task allocation for a team of aircraft launching on the carrier means obtaining the optimal launching sequence under the constraints of limited time, space, and resources [21], and it is a complex combination optimization problem in mathematical essence. The existing literature pays more attention to the operations of aircraft on the flight deck like the artificial decision support system [22], path planning algorithm [23], support scheduling model [24], and so on. As far as we know, no relevant literature has reported the task allocation method for aircraft launching on the carrier, although it is important to enhance the safety and efficiency of launching mission. Currently, the artificial heuristics approach is used to solve the task allocation problem [25]. Experienced commanders determine the launch position and order of each aircraft based on their knowledge and the environment on the flight deck. Under such a work mode, the knowledge and experience of commanders play an important role in ensuring the efficiency and safety of the launching mission. When the number of aircraft is relatively small, the commander can copy with the situation. However, it is difficult for the artificial heuristics approach to guarantee the efficiency and safety of the launching mission in the complicated environment as the number of aircraft increases. Illogical commands will lead to the congestion or collision among aircraft on deck. Therefore, a task allocation approach is necessary, which makes each aircraft reach the specified catapult with the optimal launching sequence.

This paper presents a task allocation model for a team of aircraft launching on the carrier. To make the solution easier, the task allocation problem is decomposed into two consecutive sub-tasks: catapult allocation and the launching sequence determination. Each task has its own optimization goal, and a decision-making method and a sequencing method are used to obtain the final task allocation result. The proposed model is validated by a series of comparison experiments.

\section{Problem Description and the Modeling Idea}

The aircraft goes into combat once it has received the combat mission. Figure 1 shows the general environment of flight deck. It is clear that the shape of the deck is an irregular polygon, and there are many parking aircraft. They are preparing to launch, getting recycled, or under maintenance. Four catapults are equipped to assist the aircraft in launching. The dotted lines represent the possible taxi paths between the aircraft and the catapults. The aircraft can select any possible taxi path to reach the corresponding catapult, and it is a multi-aircraft and multi-catapult launching problem. In that case, the launching position of aircraft and the launching sequence on each catapult must be determined to enhance the efficiency and safety. Note that the launch tasks belonging to two different catapults cannot be finished consecutively because the vortex flow produced by the former aircraft during the launching process will have a bad influence on the launch safety of the following aircraft. Therefore, although the aircraft launching at different catapults are permitted to taxi on the flight deck simultaneously, they have to wait to launch before the influence of vortex flow from the other catapult has disappeared.

2.1. Proposition of the Conceptual Model. Aircraft are required to complete the launching mission quickly and safely one by one following the operation flow of flight deck. However, it may lead to the congestion and collision among aircraft if the aircraft taxi at will. An optimal launch plan is necessary in advance to avoid the bad situation. The conceptual model of the task allocation problem is built in Figure 2.

The conceptual model gives a general view of solving the task allocation problem. The rest of the paper will follow these procedures and obtain the final optimal launch plan.

2.2. Framework of the Hierarchical Modeling. The task allocation is an optimization problem of decision-making and sequencing. In this paper, the hierarchical thinking is used, 


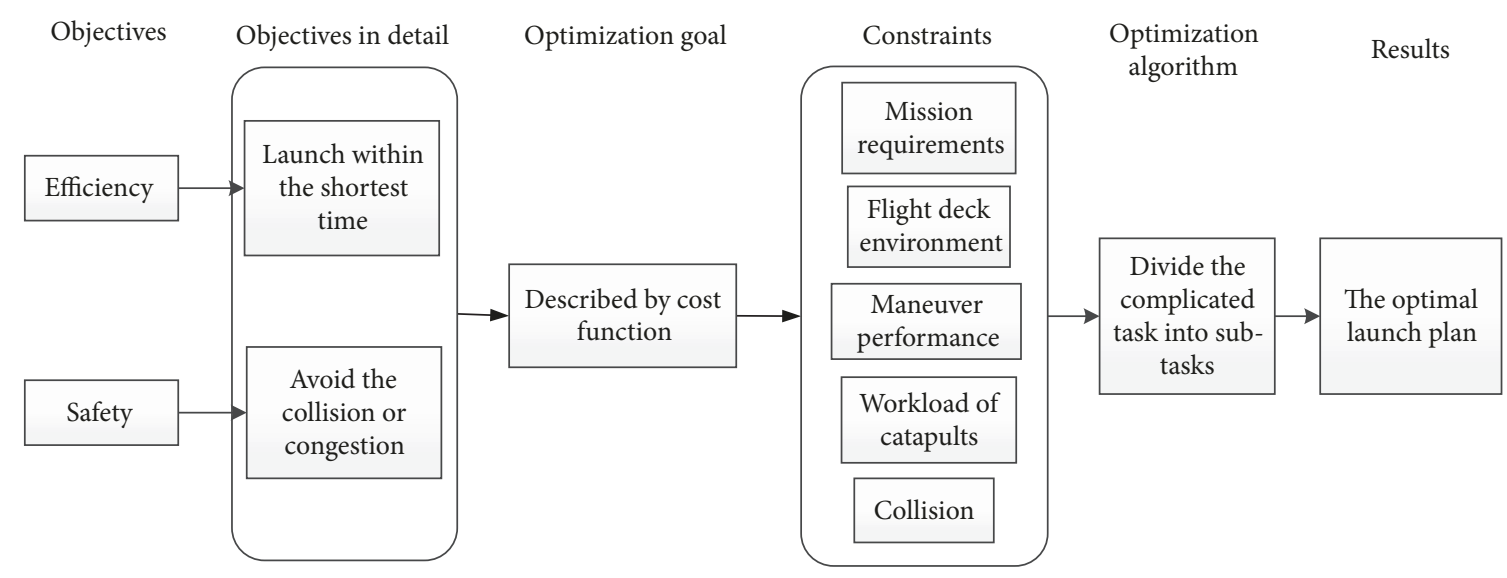

FIGURE 2: The conceptual model of launch plan determination problem.

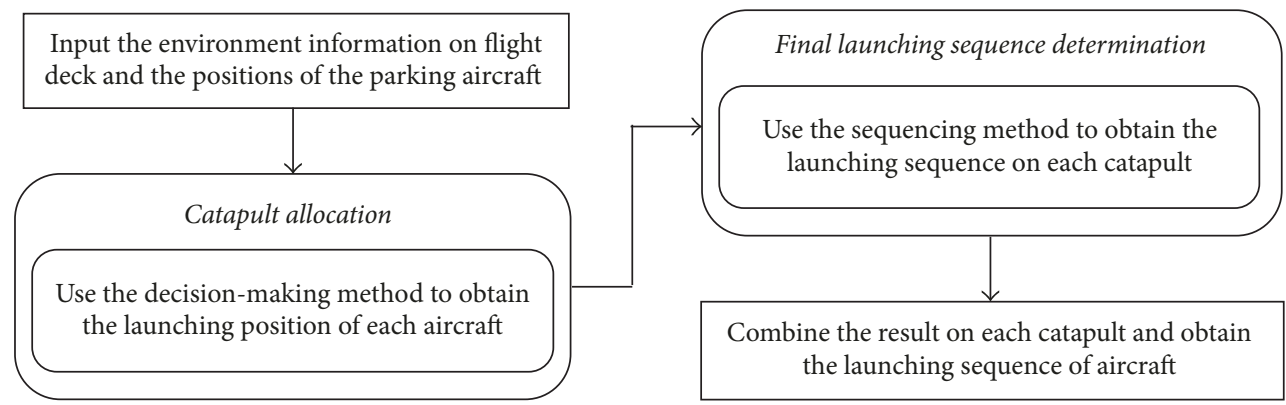

FIgURE 3: The framework of hierarchical modeling in task allocation problem.

and the task allocation problem is decomposed into two consecutive sub-tasks, that is, catapult allocation and the launching sequence determination. Different types of constraints are put into the corresponding sub-task, and each task has its own optimization goal. The hierarchical modeling reduces the complexity of problem, and the framework of hierarchical modeling is shown in Figure 3.

The results of catapult allocation are the initial conditions of the launching sequence determination, and the final optimal launch plan is the combination of launching sequence on four catapults. The hierarchical modeling identifies the goal of each sub-task and reduces the difficulty of solving the multi-aircraft and multi-catapult launching task allocation problem.

\section{Model Establishment of the Task Allocation Problem}

As mentioned above, the task allocation problem will be solved by dividing it into two consecutive sub-tasks, that is, catapult allocation and the launching sequence determination. Firstly, the general models for the two sub-tasks are established to make preparations for the further work. Then different cost functions are established in the corresponding sub-task. Specifically, the taxi time of aircraft is considered in catapult allocation, and the starting collision risk is introduced in the launching sequence determination. The optimal launch plan is obtained by optimizing the two cost functions, respectively.

3.1. Establishment of the General Models. The models of the flight deck environment and the aircraft performance are useful in the following work. Therefore, the two models are established firstly.

(1) Constraints of the Flight Deck Environment. The coordinate system $x_{d} O_{d} y_{d}$ is established at the central axis of the end flight deck, as shown in Figure $4 . V$ is defined as the set of flight deck area, and $A_{i}(i=1,2, \ldots, N)$ denotes the aircraft. $V_{i}(i=1,2, \ldots, N)$ and $C_{j}(j=1,2, \ldots, M)$ are the sets of spaces occupied by each aircraft and catapult respectively. As a footnote, the parameters $N$ and $M$ denote the number of aircraft and catapults, respectively.

The aircraft must taxi on the deck and avoid colliding with each other. The constraints can be expressed as follows:

$$
\begin{aligned}
&\left(V_{1} \cup V_{2} \cup \cdots \cup V_{N}\right) \subset V \\
& V_{s} \cap V_{t}=\varnothing \quad(s, t=1,2, \ldots, N ; s \neq t) .
\end{aligned}
$$

(2) Constraints of the Aircraft Performance. There are many kinds of performance parameters for aircraft, and here the ground maneuver performance is the most concerned. $l_{\min }$ is defined as the minimum straight path length before the 


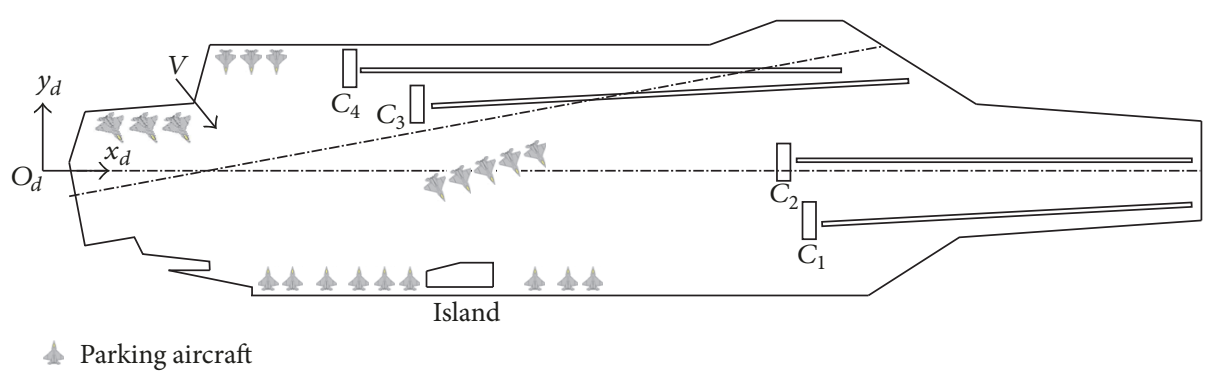

FIgURE 4: The flight deck environment and the coordinate system.

aircraft starts to change direction, and $\psi_{\max }$ is the maximum angle when the aircraft makes a turn. The matrix $F=\left[f_{i j}\right](i=1,2, \ldots, N ; j=1,2)$ is introduced to denote the ground maneuver performance, where $j=1$ and $j=2$ represent the performance of $l_{\min }$ and $\psi_{\max }$, respectively.

3.2. Solution of Catapult Allocation. The launching position of each aircraft will be determined in this task. The catapult allocation is transformed into a decision-making problem, and the launching position of each aircraft is obtained from a variety of alternative. The goal is to minimize the time aircraft taxiing on the flight deck. The decision-making model consists of the following parts.

(1) Status Set. The status set $S$ is constituted by all states of the system. Here $S$ refers to the possible pairs from $A_{i}$ to $C_{j}$, and it contains all the possible pairs of catapult allocation. The solution of catapult allocation is chosen from $S$ according to certain rules.

(2) Decision Set. The decision set $D$ is defined as the solution of catapult allocation and can be expressed by an array $L=$ $\left[l_{1}, l_{2}, \ldots, l_{i}, \ldots, l_{N}\right] . l_{i}$ is picked in $1,2, \ldots, M$ and represents the fact that $C_{l_{i}}$ is the launching position of $A_{i}$.

(3) Cost Function. The cost function is introduced to evaluate the alternatives and provide the basis for decision-making. Here the cost function matrix $T=\left[t_{i j}\right]$ is defined, where $t_{i j}$ refers to the taxi time from $A_{i}$ to $C_{j}$ calculated by the path planning method [26]. The cost function denotes the taxi time of aircraft, and aircraft prefer to reach the nearest catapult. Exceptionally, if $A_{i}$ cannot reach $C_{j}$ under the current constraints, $t_{i j}$ is set as $t_{i j}=\infty$ to express the impossible pair.

(4) Decision Rule. The decision rule $\Phi$ guarantees the feasible solution of catapult allocation and makes a balance of the workload of catapults. Without the decision rule $\Phi$, all aircraft will reach the nearest catapult, which may lead to the imbalance workload among the catapults. Some catapults are busy while others are free. The irrational catapult allocation will greatly reduce the usage efficiency of catapults, cause the congestion on the deck, and result in low efficiency of the launching mission. Therefore, the decision rule $\Phi$ is proposed to avoid the above bad situations. The decision rule $\Phi$ contains the following four items: (i) Priority will be given to the aircraft which can only reach one catapult under the current constraints.

(ii) Also, priority will be given to the catapult of which the maximum number of aircraft it can hold is less than $[N / M]$, where $[N / M]$ represents the largest integer less than $N / M$.

(iii) The rest of aircraft are preferred to be assigned to the nearest catapult, but adjustment is needed to balance the workload of each catapult. The principle is to minimize the additional time when the aircraft turns to taxi to the second nearest catapult.

In the real operation, the above three items are implemented one after another. The catapult allocation result is obtained by combining the cost function and decision rule.

Note that, in rule (i), the aircraft are given priority because there is probability that they have no catapult to select when they are far from the only catapult they can reach, which will result in an infeasible solution. Besides, in rule (ii), the catapult is given the priority in consideration of the balanced work load among each catapult. That is, the number of aircraft launching on each catapult should be approximately the same.

\subsection{Solution of the Launching Sequence Determination.} The launching sequence determination mainly aims to ensure the safety of the mission and obtain the optimal launching sequence based on the result of catapult allocation. The final optimal launch plan is the combination of the optimal launching sequence on each catapult. To obtain the optimal launching sequence on each catapult, the starting collision risk is introduced, and the optimal launching sequence is the one with the minimum collision risk.

3.3.1. Constraints of the Operation Flow. In the actual operation flow, the taxiing aircraft is prohibited to get around the immovable obstacles, because it will increase the taxi time, as shown in Figure 5.

In Figure 5, assume that $A_{1}, A_{2}, A_{3}$, and $A_{4}$ are assigned to launch at $C_{2}$ and the starting time of $A_{3}$ and $A_{4}$ must be earlier than that of $A_{1}$ and $A_{2}$ to avoid $A_{1}$ and $A_{2}$ taxiing around them. The constraint can exclude the infeasible launching sequence on each catapult.

3.3.2. Calculation of the Starting Collision Risk. The aircraft park on the flight deck regularly. Two parking modes of aircraft are introduced, as shown in Figure 6. 

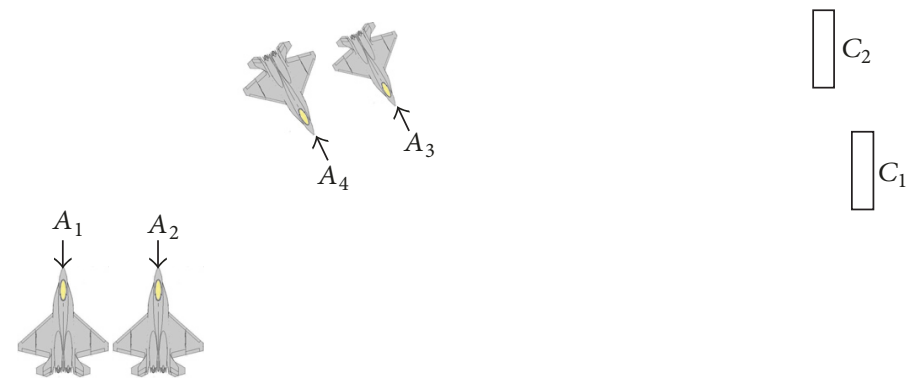

FIGURE 5: Constraints of the operation flow.

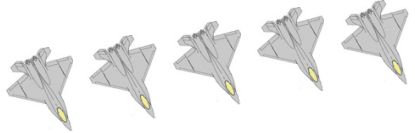

(a)

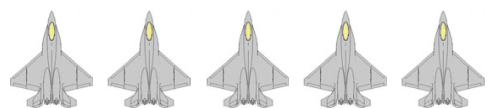

(b)

Figure 6: The parking modes of aircraft on the deck.
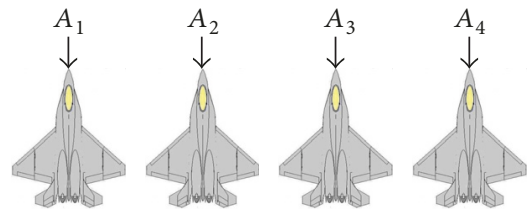

FIGURE 7: Description of the starting collision risk.

Figure 6(a) is the parking mode of aircraft in the central area of the flight deck. Aircraft are arranged tightly to save more space for the operations on fight deck. Figure 6(b) shows the parking mode of aircraft at the edge of the flight deck. They are arranged relative loosely, and their tails are all outwards the aircraft carrier to avoid the engine air flow affecting the operations on flight deck. The starting collision risk between two adjacent aircraft are defined as $r_{1}$ and $r_{2}$ $\left(r_{1}, r_{2} \in(0,1)\right)$, respectively, under the two parking modes, and $r_{1}>r_{2}$ because the aircraft arranged tighter are more likely to collide. The specific description is shown in Figure 7.

In Figure 7, assume that $A_{1}$ and $A_{4}$ are assigned to launch at $C_{1}$, and they will turn right when starting to taxi. Therefore, $A_{1}$ takes a risk of colliding with $A_{2}$ while $A_{4}$ is safe. The starting collision risk is $r_{2}$ and 0 , respectively, in the two cases.

According to Figure 7, we can express the average starting collision risk of each feasible launching sequence on $C_{j}$ as follows:

$$
R_{j h}=\frac{\left(\sum_{k=1}^{N_{j}} P_{k}\right)}{N_{j}},
$$

where $h=1,2, \ldots, A_{N_{j}}^{N_{j}}\left(A_{N_{j}}^{N_{j}}\right.$ is the number of permutations which denotes all the launching sequence on $C_{j}$ ); $N_{j}$ is the number of aircraft launching at $C_{j} ; P_{k}\left(P_{k} \in\left\{0, r_{1}, r_{2}\right\}\right)$ is the starting collision risk; and $R_{j h}$ is the average starting collision risk of each launching sequence on $C_{j}$. Note that if a launching sequence is infeasible, that is, the constraint set in Section 3.3.1 is violated, $R_{j h}$ is set as $R_{j h}=\infty$. The launching sequence corresponding to the minimum value of $R_{j h}$ is the optimal launching sequence on the catapult.

3.3.3. The Launching Sequence Determination. The launching sequence determination is the combination of the optimal launching sequence on each catapult. The optimal launching sequence on $C_{j}$ can be calculated in

$$
\mathrm{OLS}_{j}=\min \left\{R_{j 1}, R_{j 2}, \ldots, R_{j A_{N_{j}}^{N_{j}}}\right\},
$$

where OLS ${ }_{j}$ is the optimal launching sequence on $C_{j}$, that is, the launching sequence with the minimum starting collision risk. The final launching sequence for the whole team of aircraft can be expressed in

$$
\mathrm{LSA}=\mathrm{OLS}_{1} \cup \mathrm{OLS}_{2} \cup \mathrm{OLS}_{3} \cup \mathrm{OLS}_{4} .
$$

LSA denotes the launching sequence of aircraft. It is a union of optimal launching sequence on each catapult. 


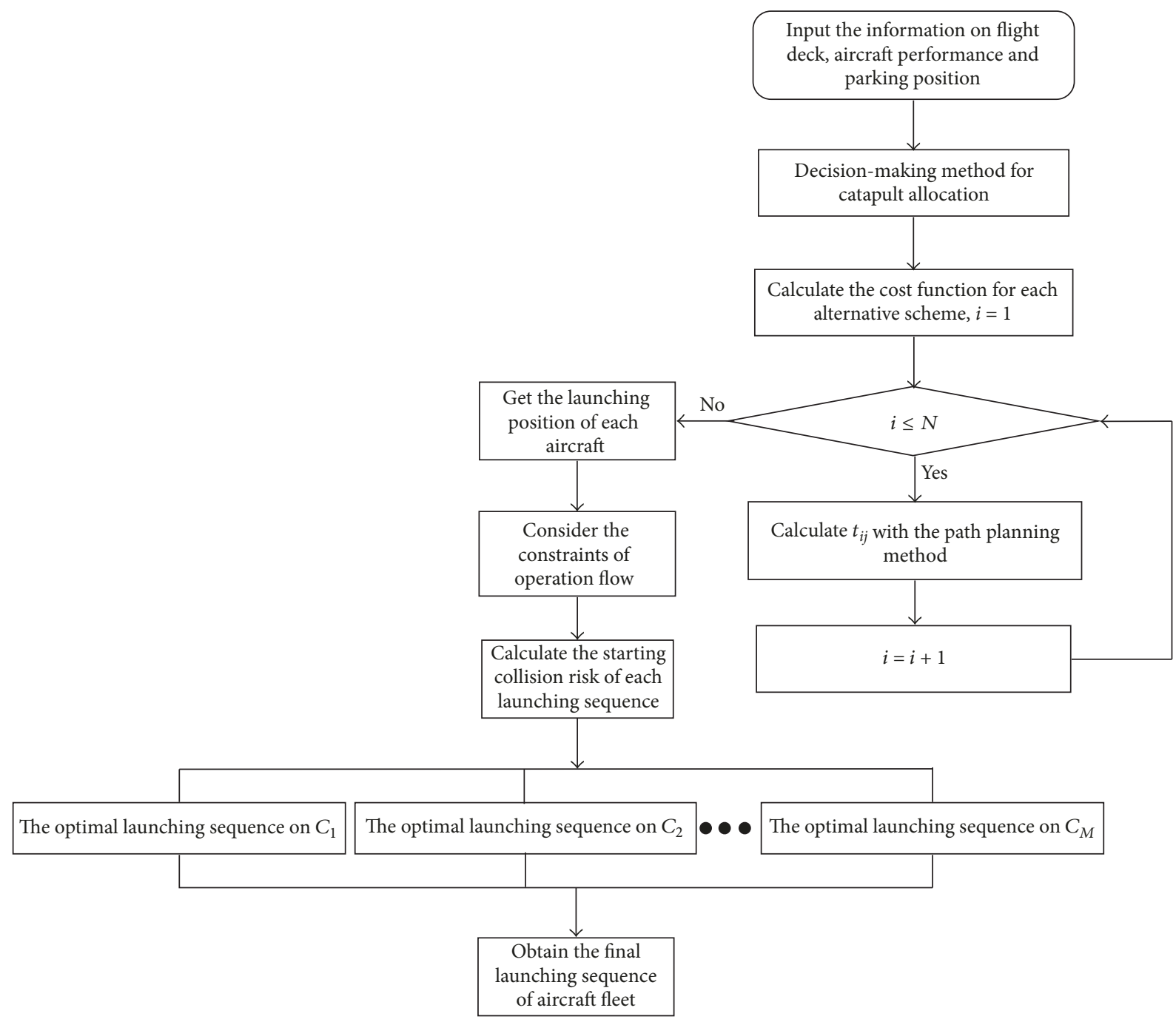

FIGURE 8: Flow of the task allocation method.

3.4. Procedure of the Task Allocation Method. The previous chapters introduce the task allocation method in detail. A flow chart is needed to make a better understanding of the method, as shown in Figure 8.

\section{Experimental Studies}

In the actual combat circumstance, the average number of aircraft for mission use is about 20 on the flight deck of the Nimitz-class aircraft carrier. The launch plan is obtained under the artificial heuristics approach and the proposed task allocation method, respectively. Besides, the brute force approach is also used, and the comparisons among the three methods are performed. It demonstrates the validity of the proposed method and the limitation of other two approaches.

4.1. Model of the Experiment and the Parameters Settings. The model of the Nimitz-class aircraft carrier and the parking position of each aircraft are presented in Figure 9. In addition, the parameters settings are shown in Table 1.
In Figure 9, the rest aircraft are under maintenance and not taken into consideration in task allocation problem.

4.2. The Results with Artificial Heuristics Approach. In the experiment, the experienced commander working on aircraft carrier is invited to give the launch plan. The basis of artificial heuristics approach makes references to the expert user heuristics in [25] and is listed in Figure 10.

According to the above basis, the commander gives the launch plan, as shown in Figure 11.

It turns out that the commander makes the workload of catapults balanced and tries to reduce the collision risk. However, the ground maneuver performance of aircraft is ignored, and the aircraft may be unable to reach the specified catapult directly. As a result, the taxi time will become longer due to the detour, reverse, or congestion in taxiing.

4.3. The Results with the Task Allocation Method. The artificial heuristics approach has exposed its defects in the feasibility of taxi path. Next, the task allocation is used to obtain the launch plan. 


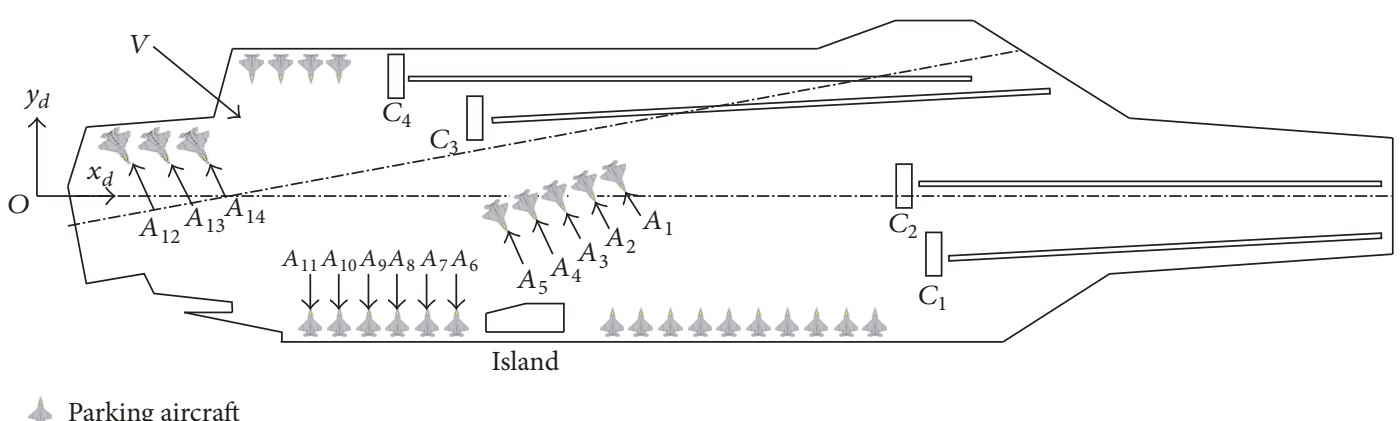

FIGURE 9: The experiment model and the parking position of aircraft.

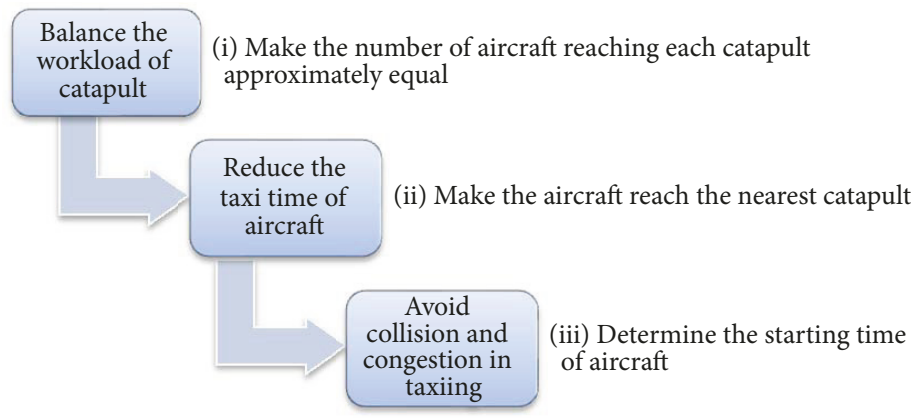

FIGURE 10: Basis of the artificial heuristics approach.

TABLE 1: Parameters settings in the experiment.

\begin{tabular}{lccc}
\hline Aircraft & $\begin{array}{c}\text { Coordinates of the } \\
\text { parking stand }\end{array}$ & Catapult & $\begin{array}{c}\text { Coordinates of the } \\
\text { catapult }\end{array}$ \\
\hline$A_{1}$ & $(155.3,7.9)$ & $C_{1}$ & $(223.6,-16.1)$ \\
$A_{2}$ & $(144.3,4.0)$ & $C_{2}$ & $(219.5,4.1)$ \\
$A_{3}$ & $(133.3,0)$ & $C_{3}$ & $(119.9,18.9)$ \\
$A_{4}$ & $(122.3,-4.0)$ & $C_{4}$ & $(103.8,32.3)$ \\
$A_{5}$ & $(111.3,-7.9)$ & & \\
$A_{6}$ & $(125.0,-37.7)$ & & \\
$A_{7}$ & $(113.0,-37.7)$ & & \\
$A_{8}$ & $(101.0,-37.7)$ & & \\
$A_{9}$ & $(89.0,-37.7)$ & & \\
$A_{10}$ & $(77.0,-37.7)$ & & \\
$A_{11}$ & $(65.0,-37.7)$ & & \\
$A_{12}$ & $(11.4,16.7)$ & & \\
$A_{13}$ & $(22.4,17.5)$ & & \\
$A_{14}$ & $(33.4,18.2)$ & & \\
\hline
\end{tabular}

4.3.1. Results of the Catapult Allocation. We input the information on the flight deck environment, the aircraft parking, and the matrix of ground maneuver performance $F$. Multiple paths are planned for each aircraft reaching different catapults [26]. 3 cases with different constraints are given to explain their influences on the results, as represented in Table 2.

Figures 12-14 show the path planning results in the 3 cases.

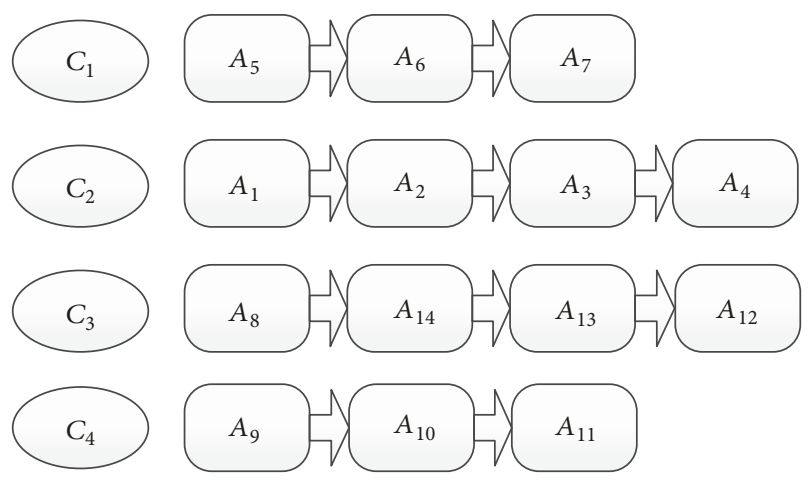

FIGURE 11: The launch plan with the artificial heuristics approach.

Tables 3-5 conclude the results of Figures 12-14. " $\sqrt{ }$ " denotes that $A_{i}(i=1,2, \ldots, 14)$ can reach $C_{j}(j=1,2,3,4)$ under such constraints, and " $x$ " denotes the opposite. We assume the aircraft taxies with the uniform motion of $5 \mathrm{ft} / \mathrm{s}$, and the taxi time of each pair from $A_{i}$ to $C_{j}$ in case (3) are shown in Table 5.

In Table 3, none of aircraft can reach $C_{4}$ due to the tough constraint of ground maneuver performance, and it makes $C_{4}$ free. On the other hand, weak constraints result in different pairs from $A_{i}$ to $C_{j}$, as shown in Table 4 . Therefore, proper constraints are needed to guarantee the rationality of results, and we continue the experiment with the parameters settings in case (3).

In Table $5, A_{1}-A_{6}$ can only reach $C_{1}$ and $C_{2}$ under the constraints. Therefore, they are given priority according to the 
TABLE 2: Settings of the different constraints and explanations.

Serial number and explanation Parameters setting

(1) Take into account the constraint of deck shape and give tough constraint to the ground maneuver performance of aircraft.

the matrix of ground maneuver performance $F_{1}$ : $f_{i 1}=25 \mathrm{ft}, i=1,2, \ldots, N$

(2) Take no account of the deck shape and give weak constraint to the ground maneuver performance of aircraft.

the matrix of ground maneuver performance $F_{2}$ :

$f_{i 2}=30^{\circ}, i=1,2, \ldots, N$

(3) Take into account the constraint of deck shape and give

reasonable constraint to the ground maneuver performance the matrix of ground maneuver performance $F_{3}$ : of aircraft.

$f_{i 1}=10 \mathrm{ft}, i=1,2, \ldots, N$

$f_{i 2}=180^{\circ}, i=1,2, \ldots, N$

$f_{i 1}=10 \mathrm{ft}, i=1,2, \ldots, N$

$f_{i 2}=70^{\circ}, i=1,2, \ldots, N$

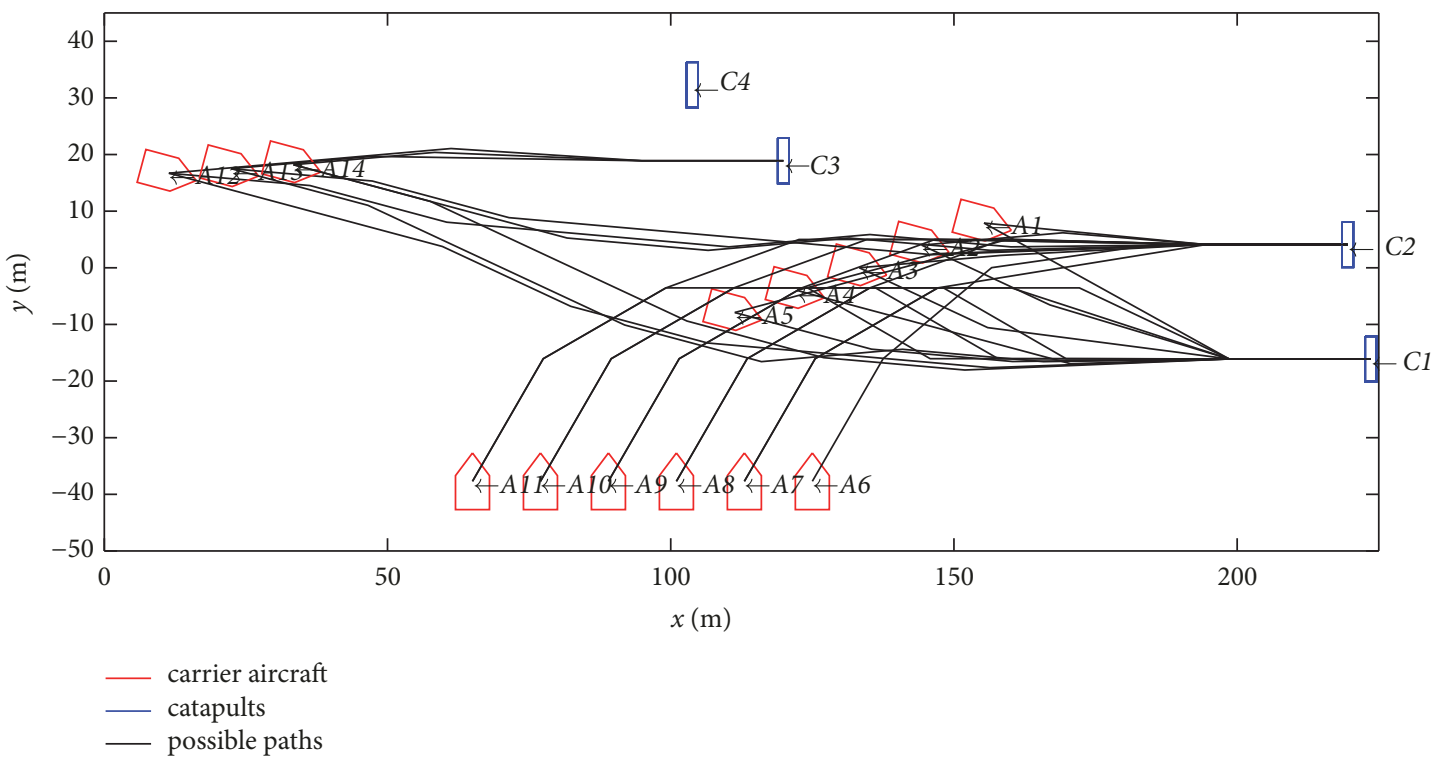

Figure 12: Path planning results of case (1).

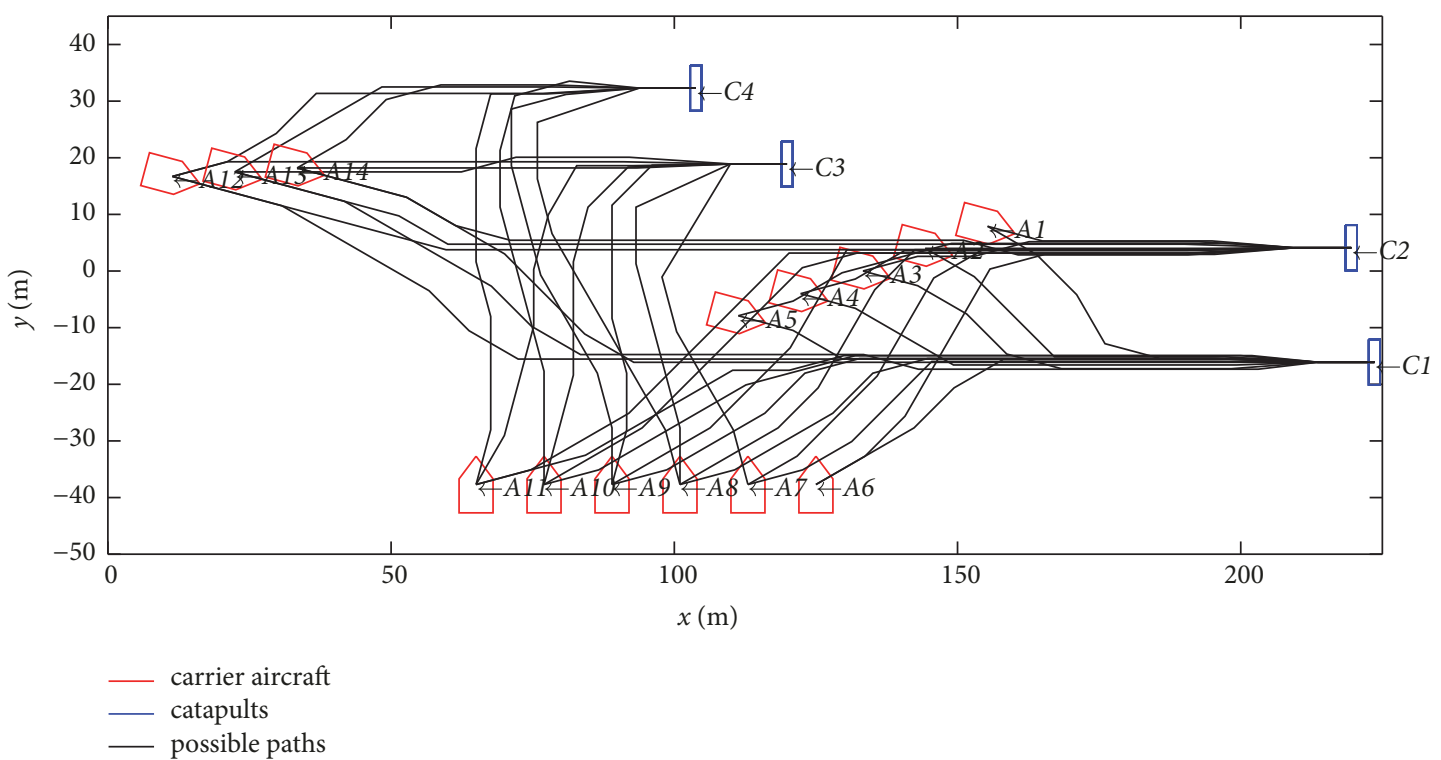

FIGURE 13: Path planning results of case (2). 
TABLE 3: The results of case (1).

\begin{tabular}{ccccccccccccccc}
\hline & $A_{1}$ & $A_{2}$ & $A_{3}$ & $A_{4}$ & $A_{5}$ & $A_{6}$ & $A_{7}$ & $A_{8}$ & $A_{9}$ & $A_{10}$ & $A_{11}$ & $A_{12}$ & $A_{13}$ & $A_{14}$ \\
\hline$C_{1}$ & $\sqrt{ }$ & $\sqrt{ }$ & $\sqrt{ }$ & $\sqrt{ }$ & $\sqrt{ }$ & $\sqrt{ }$ & $\sqrt{ }$ & $\sqrt{ }$ & $\sqrt{ }$ & $\sqrt{ }$ & $\sqrt{ }$ & $\sqrt{ }$ & $\sqrt{ }$ & $\sqrt{ }$ \\
$C_{2}$ & $\sqrt{ }$ & $\sqrt{ }$ & $\sqrt{ }$ & $\sqrt{ }$ & $\sqrt{ }$ & $\sqrt{ }$ & $\sqrt{ }$ & $\sqrt{ }$ & $\sqrt{ }$ & $\sqrt{ }$ & $\sqrt{ }$ & $\sqrt{ }$ & $\sqrt{ }$ & $\sqrt{ }$ \\
$C_{3}$ & $\times$ & $\times$ & $\times$ & $\times$ & $\times$ & $\times$ & $\times$ & $\times$ & $\times$ & $\times$ & $\times$ & $\sqrt{ }$ & $\sqrt{ }$ & $\sqrt{ }$ \\
$C_{4}$ & $\times$ & $\times$ & $\times$ & $\times$ & $\times$ & $\times$ & $\times$ & $\times$ & $\times$ & $\times$ & $\times$ & $\times$ & $\times$ & $\times$ \\
\hline
\end{tabular}

TABLE 4: The results of case (2).

\begin{tabular}{cccccccccccccccc}
\hline & $A_{1}$ & $A_{2}$ & $A_{3}$ & $A_{4}$ & $A_{5}$ & $A_{6}$ & $A_{7}$ & $A_{8}$ & $A_{9}$ & $A_{10}$ & $A_{11}$ & $A_{12}$ & $A_{13}$ & $A_{14}$ \\
\hline$C_{1}$ & $\sqrt{ }$ & $\sqrt{ }$ & $\sqrt{ }$ & $\sqrt{ }$ & $\sqrt{ }$ & $\sqrt{ }$ & $\sqrt{ }$ & $\sqrt{ }$ & $\sqrt{ }$ & $\sqrt{ }$ & $\sqrt{ }$ & $\sqrt{ }$ & $\sqrt{ }$ & $\sqrt{ }$ \\
$C_{2}$ & $\sqrt{ }$ & $\sqrt{ }$ & $\sqrt{ }$ & $\sqrt{ }$ & $\sqrt{ }$ & $\sqrt{ }$ & $\sqrt{ }$ & $\sqrt{ }$ & $\sqrt{ }$ & $\sqrt{ }$ & $\sqrt{ }$ & $\sqrt{ }$ & $\sqrt{ }$ & $\sqrt{ }$ \\
$C_{3}$ & $\times$ & $\times$ & $\times$ & $\times$ & $\times$ & $\times$ & $\sqrt{ }$ & $\sqrt{ }$ & $\sqrt{ }$ & $\sqrt{ }$ & $\sqrt{ }$ & $\sqrt{ }$ & $\sqrt{ }$ & $\sqrt{ }$ \\
$C_{4}$ & $\times$ & $\times$ & $\times$ & $\times$ & $\times$ & $\times$ & $\times$ & $\sqrt{ }$ & $\sqrt{ }$ & $\sqrt{ }$ & $\sqrt{ }$ & $\sqrt{ }$ & $\sqrt{ }$ & $\sqrt{ }$ \\
\hline
\end{tabular}

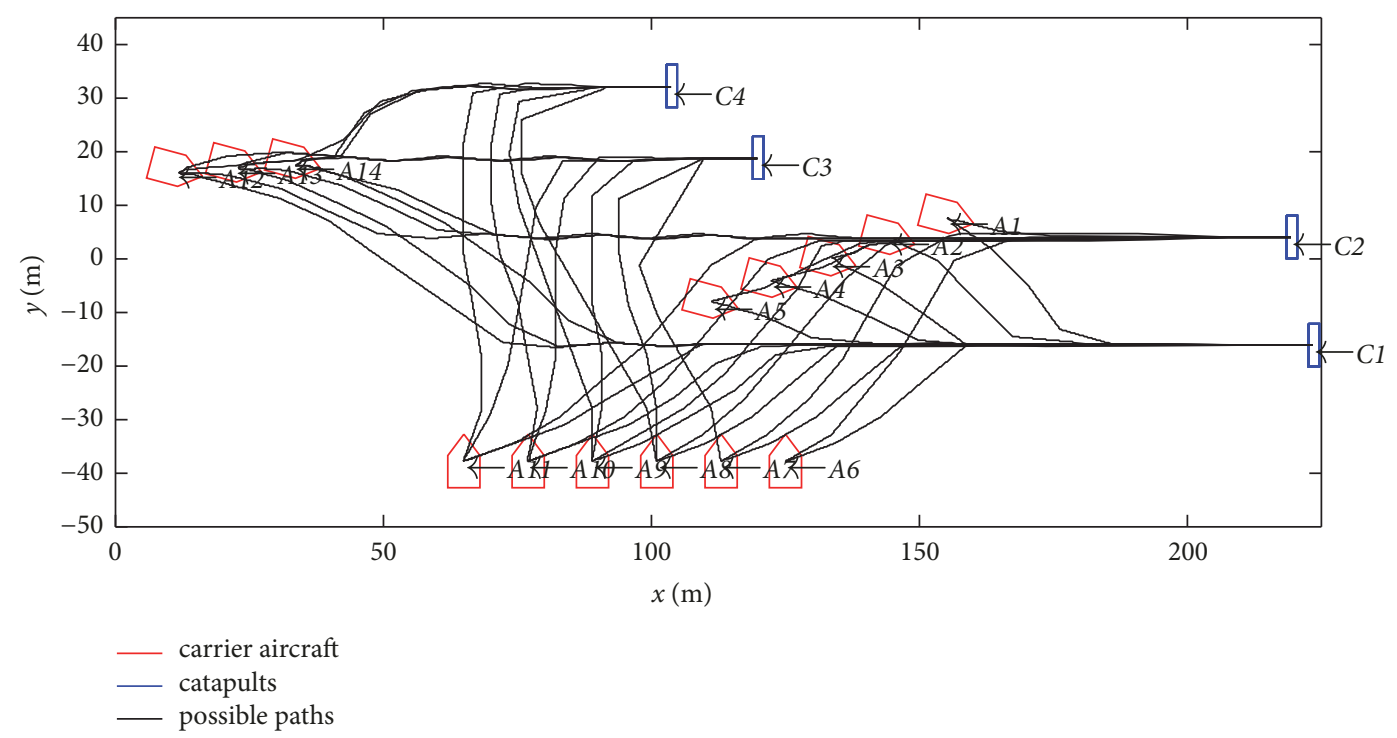

FIGURE 14: Path planning results of case (3).

TABLE 5: Taxi time of each pair in case (3).

\begin{tabular}{lcccc}
\hline & $C_{1}$ & $C_{2}$ & $C_{3}$ & $C_{4}$ \\
\hline$A_{1}$ & $15.5 \mathrm{~s}$ & $12.9 \mathrm{~s}$ & $\times$ & $\times$ \\
$A_{2}$ & $17.2 \mathrm{~s}$ & $15.1 \mathrm{~s}$ & $\times$ & $\times$ \\
$A_{3}$ & $19.0 \mathrm{~s}$ & $17.3 \mathrm{~s}$ & $\times$ & $\times$ \\
$A_{4}$ & $20.7 \mathrm{~s}$ & $19.7 \mathrm{~s}$ & $\times$ & $\times$ \\
$A_{5}$ & $22.8 \mathrm{~s}$ & $22.1 \mathrm{~s}$ & $\times$ & $\times$ \\
$A_{6}$ & $21.0 \mathrm{~s}$ & $22.7 \mathrm{~s}$ & $\times$ & $\times$ \\
$A_{7}$ & $23.4 \mathrm{~s}$ & $25.0 \mathrm{~s}$ & $14.7 \mathrm{~s}$ & $\times$ \\
$A_{8}$ & $25.8 \mathrm{~s}$ & $27.1 \mathrm{~s}$ & $15.5 \mathrm{~s}$ & $19.8 \mathrm{~s}$ \\
$A_{9}$ & $28.1 \mathrm{~s}$ & $29.6 \mathrm{~s}$ & $16.7 \mathrm{~s}$ & $19.8 \mathrm{~s}$ \\
$A_{10}$ & $30.4 \mathrm{~s}$ & $31.6 \mathrm{~s}$ & $17.9 \mathrm{~s}$ & $20.4 \mathrm{~s}$ \\
$A_{11}$ & $32.8 \mathrm{~s}$ & $33.9 \mathrm{~s}$ & $19.3 \mathrm{~s}$ & $21.5 \mathrm{~s}$ \\
$A_{12}$ & $44.3 \mathrm{~s}$ & $42.2 \mathrm{~s}$ & $21.8 \mathrm{~s}$ & $19.6 \mathrm{~s}$ \\
$A_{13}$ & $42.3 \mathrm{~s}$ & $40.0 \mathrm{~s}$ & $19.6 \mathrm{~s}$ & $17.4 \mathrm{~s}$ \\
$A_{14}$ & $40.1 \mathrm{~s}$ & $37.8 \mathrm{~s}$ & $17.3 \mathrm{~s}$ & $14.9 \mathrm{~s}$ \\
\hline
\end{tabular}

decision rule (i). Note that only $A_{6}$ reaches $C_{1}$ with less taxi time than $C_{2}$ among $A_{1}-A_{6}$. If the decision is made based on the cost function merely, $C_{1}$ and $C_{2}$ will have imbalanced workload. Therefore, an adjustment is needed according to the decision rule (iii). The additional time when $A_{1}-A_{5}$ turn to taxi to $C_{1}$ is listed in Figure 15.

$A_{4}$ and $A_{5}$ have less addition time, and they are assigned to launch at $C_{1}$. The launching position of the rest unassigned aircraft can also be determined by the decision-making method. Finally, the decision set $D$ is obtained, and it is denoted with the array $L$ as follows:

$$
L=\left[\begin{array}{llllllllllllll}
2 & 2 & 2 & 1 & 1 & 1 & 3 & 3 & 3 & 3 & 4 & 4 & 4 & 4
\end{array}\right] .
$$

To make it clearer, we represent the above results in Figure 16.

Comparing with the artificial heuristics approach, the task allocation method takes into account the ground maneuver performance of aircraft and gets the taxi time of each 


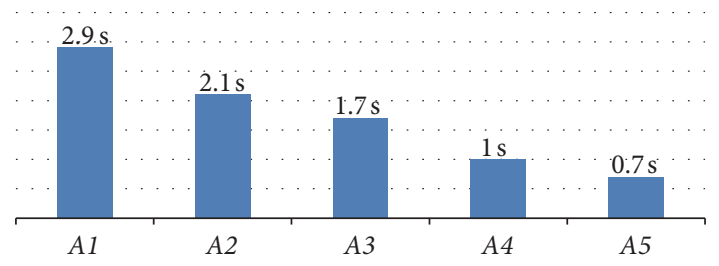

FIGURE 15: The additional time of each aircraft.

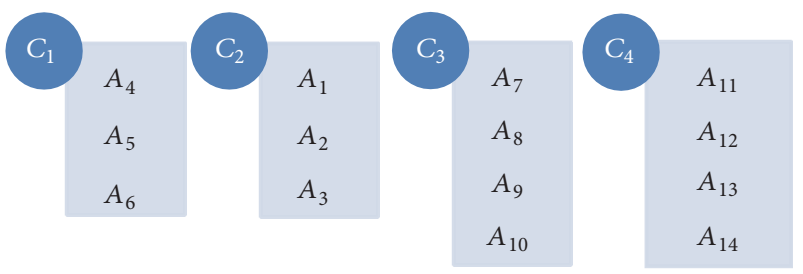

FIGURE 16: Results of the catapult allocation.

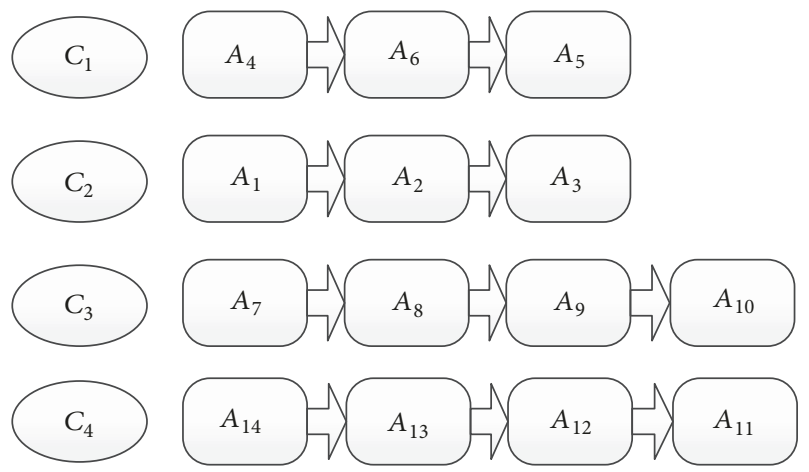

FIGURE 17: The launch plan with the task allocation method.

aircraft with the path planning algorithm. It gives a more scientific and comprehensive consideration instead of relying on the knowledge and experience of commander.

4.3.2. Results of the Launching Sequence Determination. The experiment continues based on the results of the catapult allocation. Assume that $r_{1}=0.5$ and $r_{2}=0.3$, and the average starting collision risk of each feasible launching sequence is calculated, as shown in Table 6. The launch plan is shown in Figure 17.

The quantitative collision risk is given in the task allocation method compared with the artificial heuristics approach, and the safety of launching is guaranteed. Furthermore, more quantitative comparison study will be conducted in the next chapter to indicate the superiority of the proposed task allocation method.

4.4. Comparisons among Different Methods. Here the brute force approach is introduced to solve the task allocation problem. The brute force approach is a very time-consuming algorithm that lists all possibilities and selects the best. The optimal launch plan regarding efficiency and safety are shown in Figures 18 and 19, respectively.

\section{TABLE 6}

(a) The average starting collision risk of feasible launching sequence on $C_{1}$

\begin{tabular}{llc}
\hline Catapult allocation & \multicolumn{1}{c}{$A_{4}, A_{5}, A_{6}$} \\
& $A_{4} \rightarrow A_{5} \rightarrow A_{6}$ & Risk \\
\hline Feasible launching & $A_{4} \rightarrow A_{6} \rightarrow A_{5}$ & 0.17 \\
sequence and & $A_{5} \rightarrow A_{4} \rightarrow A_{6}$ & 0.17 \\
average starting & $A_{5} \rightarrow A_{6} \rightarrow A_{4}$ & 0.33 \\
collision risk & $A_{6} \rightarrow A_{4} \rightarrow A_{5}$ & 0.33 \\
& $A_{6} \rightarrow A_{5} \rightarrow A_{4}$ & 0.17 \\
\hline
\end{tabular}

(b) The average starting collision risk of feasible launching sequence on $C_{2}$

\begin{tabular}{llc}
\hline Catapult allocation & \multicolumn{1}{c}{$A_{1}, A_{2}, A_{3}$} \\
& $A_{1} \rightarrow A_{2} \rightarrow A_{3}$ & Risk \\
\hline Feasible launching & $A_{1} \rightarrow A_{3} \rightarrow A_{2}$ & 0 \\
sequence and & $A_{2} \rightarrow A_{1} \rightarrow A_{3}$ & 0.17 \\
average starting & $A_{2} \rightarrow A_{3} \rightarrow A_{1}$ & 0.17 \\
collision risk & $A_{3} \rightarrow A_{1} \rightarrow A_{2}$ & 0.17 \\
& $A_{3} \rightarrow A_{2} \rightarrow A_{1}$ & 0.17 \\
\hline
\end{tabular}

(c) The launching sequence with the minimum average starting collision risk on $C_{3}$ and $C_{4}$

\begin{tabular}{lcc}
\hline Catapult & Sequence & Risk \\
\hline$C_{3}$ & $A_{7} \rightarrow A_{8} \rightarrow A_{9} \rightarrow A_{10}$ & 0.075 \\
\hline$A_{14} \rightarrow A_{13} \rightarrow A_{12} \rightarrow A_{11}$ & \\
$C_{4}$ & $A_{14} \rightarrow A_{13} \rightarrow A_{11} \rightarrow A_{12}$ & 0.075 \\
& $A_{14} \rightarrow A_{11} \rightarrow A_{13} \rightarrow A_{12}$ & \\
& $A_{11} \rightarrow A_{14} \rightarrow A_{13} \rightarrow A_{12}$ & \\
\hline
\end{tabular}

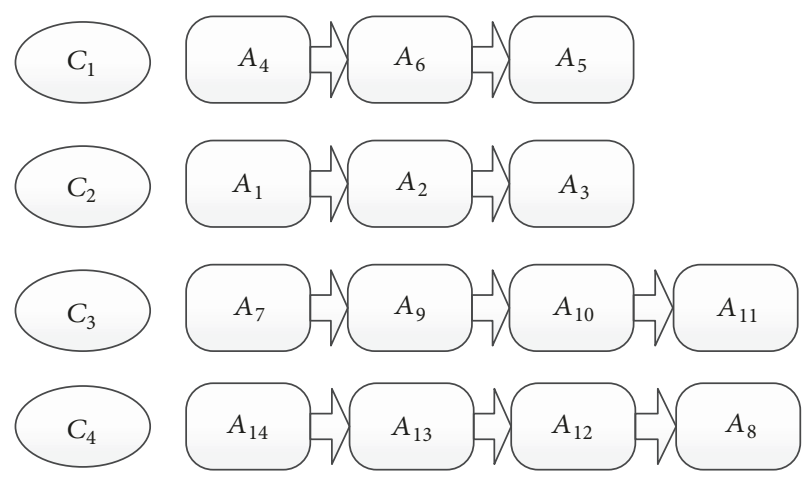

FIGURE 18: The launch plan with the shortest mission time under the brute force approach.

Comparisons among the three methods are performed to explain the validity of the proposed task allocation method. Assume that it takes $120 \mathrm{~s}$ for the aircraft to make preparations on the catapult before it launches. The mission time and the average starting collision risk can be calculated according to the given launch plan. The results with different methods are presented in Table 7. 
TABLE 7: The results with different methods.

\begin{tabular}{lcc}
\hline & Mission time & Collision risk \\
\hline Artificial heuristics approach & $454.2 \mathrm{~s}$ & 0.345 \\
Task allocation method & $433.4 \mathrm{~s}$ & 0.32 \\
Brute force approach & & $431.7 \mathrm{~s}$ \\
With the shortest mission time & $453 \mathrm{~s}$ & 0.395 \\
With the minimum collision risk & 0.225 \\
\hline
\end{tabular}

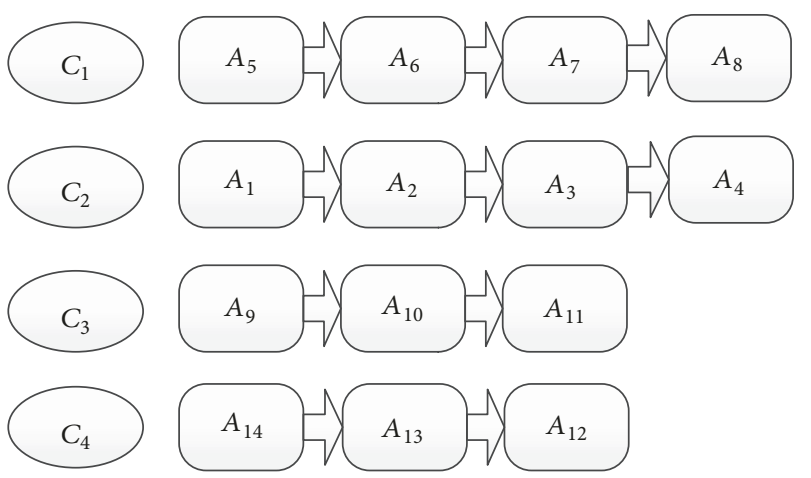

FIGURE 19: The launch plan with the minimum average starting collision risk under the brute force approach.

The experiment results demonstrate that the efficiency and safety of the mission are improved with the task allocation method compared to the artificial heuristics approach, because the ground maneuver performance and the collision risk of aircraft are considered quantitatively in the task allocation method. Although the efficiency is slightly improved with the brute force approach, the average starting collision risk is the maximum among the three methods. In addition, the brute force approach can minimize the average starting collision risk at the cost of reducing the efficiency. In general, the proposed task allocation method gives a comprehensive consideration of the efficiency and safety of the launching mission, and the brute force approach can only choose either the average starting collision risk or the efficiency as an objective function. Note that, the launch plan is generated offline in this paper, so the calculation time of each method is not the main concern. In the brute force approach, the calculation time will grow exponentially with the increasing number of aircraft. This is because the number of possible solutions for the brute force approach will be $4^{n}$ ( $n$ and 4 are the number of aircraft and catapults, resp.). As for the artificial heuristics approach, a large number of aircraft also make it overwhelmed.

\section{Conclusion}

The task allocation problem for a team of aircraft launching on the carrier is addressed in this paper. The goal is to improve the efficiency and safety level of the launching mission. Firstly, the conceptual model is built to give a general idea of solving the problem. Then the problem is decomposed into consecutive sub-tasks, that is, catapult allocation and the launching sequence determination. Thirdly, the proposed method is validated using the setups of the Nimitz-class aircraft carrier. The proposed task allocation method is used to solve the task allocation problem, and is compared to the artificial heuristics approach and the brute force approach.

Simulation results show that the proposed task allocation method has better performance than the artificial heuristics approach and the brute force approach especially when considering the balance of the efficiency and safety. The proposed method is better than the artificial heuristics approach mainly due to the fact that the ground maneuver performance of aircraft is ignored in the artificial heuristics approach. In practice, the aircraft may be unable to reach the specified catapult directly because the taxi time will become longer due to detour, reverse, or congestion in taxiing. Though the proposed method has slightly lower efficiency than the brute force approach, the former results in lowest average starting collision risk compared to the other two methods. Note that the brute force approach can only choose either the average starting collision risk or the efficiency as an objective function.

\section{Data Availability}

The data used to support the findings of this study are available from the corresponding author upon request.

\section{Conflicts of Interest}

The authors declare that there are no conflicts of interest regarding the publication of this paper.

\section{Acknowledgments}

This research work is financially supported by the Fundamental Research Funds for the Central Universities with the Project Reference no. 106112016 CDJRC000107.

\section{References}

[1] D. Shapley, "Aircraft Carriers: Pentagon Split on Issue That Will Shape Navy's Future," Science, vol. 190, no. 4210, pp. 130-134, 1975.

[2] Y. Wu, L. Sun, and X. Qu, "A sequencing model for a team of aircraft landing on the carrier," Aerospace Science and Technology, vol. 54, pp. 72-87, 2016.

[3] G. I. Rochlin, T. R. La Porte, and K. H. Roberts, "The selfdesigning high-reliability organization: Aircraft carrier flight 
operations at sea," Naval War College Review, vol. 40, no. 4, pp. 76-90, 1987.

[4] K. H. Roberts, "Some Characteristics of One Type of High Reliability Organization," Organization Science, vol. 1, no. 2, pp. 160-176, 1990.

[5] S. Newman, "Shipborne aviation: An introduction," Proceedings of the Institution of Mechanical Engineers, Part G: Journal of Aerospace Engineering, vol. 219, no. 5, pp. 363-368, 2005.

[6] C. Wang, J. Li, N. Jing, J. Wang, and H. Chen, "A distributed cooperative dynamic task planning algorithm for multiple satellites based on multi-agent hybrid learning," Chinese Journal of Aeronautics, vol. 24, no. 4, pp. 493-505, 2011.

[7] J. Dou, C. Chen, and P. Yang, "Genetic Scheduling and Reinforcement Learning in Multirobot Systems for Intelligent Warehouses," Mathematical Problems in Engineering, vol. 2015, Article ID 597956, 2015.

[8] H. Luo, Y. Niu, M. Zhu, X. Hu, and H. Ma, "Optimization of Pesticide Spraying Tasks via Multi-UAVs Using Genetic Algorithm," Mathematical Problems in Engineering, vol. 2017, pp. 1-16, 2017.

[9] H. Bui, X. Han, S. Mandal, K. R. Pattipati, and D. L. Kleinman, "Optimization-based decision support algorithms for a teamin-the-loop planning experiment," in Proceedings of the 2009 IEEE International Conference on Systems, Man and Cybernetics, SMC 2009, pp. 4684-4689, usa, October 2009.

[10] Y. Wu, Z. Huang, Y. Li, and Z. Wang, "Modeling Multioperator Multi-UAV Operator Attention Allocation Problem Based on Maximizing the Global Reward," Mathematical Problems in Engineering, vol. 2016, Article ID 1825134, 2016.

[11] I. Anagnostakis, J. Clarke, D. Bohme, and U. Volckers, "Runway operations planning and control: Sequencing and scheduling," Journal of Aircraft, vol. 38, no. 6, pp. 988-996, 2001.

[12] I. A. McManus and R. A. Walker, "Multidisciplinary approach to intelligent unmanned-airborne-vehicles mission planning," Journal of Aircraft, vol. 43, no. 2, pp. 318-335, 2006.

[13] S. Y. P. Chien, L. Q. Xue, and M. Palakal, "Task planning for a mobile robot in an indoor environment using object-oriented domain information," IEEE Transactions on Systems, Man, and Cybernetics, Part B: Cybernetics, vol. 27, no. 6, pp. 1007-1016, 1997.

[14] S. Bagchi, G. Biswas, and K. Kawamura, "Task planning under uncertainty using a spreading activation network," IEEE Transactions on Systems, Man and Cybernetics, Part A: Systems and Humans, vol. 30, no. 6, pp. 639-650, 2000.

[15] Y. Eun and H. Bang, "Cooperative task assignment/Path planning of multiple unmanned aerial vehicles using genetic algorithms," Journal of Aircraft, vol. 46, no. 1, pp. 338-343, 2009.

[16] W. Zhang, M. Kamgarpour, D. Sun, and C. J. Tomlin, "A hierarchical flight planning framework for air traffic management," Proceedings of the IEEE, vol. 100, no. 1, pp. 179-194, 2012.

[17] T. Wei, X. Qu, and L. Wang, "Hierarchical mission planning for multiple vehicles airdrop operation," Aircraft Engineering and Aerospace Technology, vol. 83, no. 5, pp. 315-323, 2011.

[18] G. Gupta, W. Malik, and Y. C. Jung, "A mixed integer linear program for airport departure scheduling," in Proceedings of the 9th AIAA Aviation Technology, Integration and Operations (ATIO) Conference, Aircraft Noise and Emissions Reduction Symposium (ANERS), usa, September 2009.

[19] J. A. Bennell, M. Mesgarpour, and C. N. Potts, "Airport runway scheduling," Annals of Operations Research, vol. 204, no. 1, pp. 249-270, 2013.
[20] H. Zhou and X. Jiang, "Multirunway Optimization Schedule of Airport Based on Improved Genetic Algorithm by Dynamical Time Window," Mathematical Problems in Engineering, vol. 2015, Article ID 854372, 2015.

[21] D.-W. Yoo, C.-H. Lee, M.-J. Tahk, and H.-L. Choi, "Optimal resource management algorithm for unmanned aerial vehicle missions in hostile territories," Proceedings of the Institution of Mechanical Engineers, Part G: Journal of Aerospace Engineering, vol. 228, no. 12, pp. 2157-2167, 2014.

[22] J. C. Ryan, M. L. Cummings, N. Roy, A. Banerjee, and A. Schulte, "Designing an interactive local and global decision support system for aircraft carrier deck scheduling," in Proceedings of the AIAA Infotech at Aerospace Conference and Exhibit, St. Louis, Miss, USA, March 2011.

[23] W. Han, W. Si, D. Ding, and Y. Song, "Multi-routes dynamic planning on deck of carrier plane based on clustering PSO," Beijing Hangkong Hangtian Daxue Xuebao/Journal of Beijing University of Aeronautics and Astronautics, vol. 39, no. 5, pp. 610-614, 2013.

[24] Q. Feng, S. Li, and B. Sun, "A multi-agent based intelligent configuration method for aircraft fleet maintenance personnel," Chinese Journal of Aeronautics, vol. 27, no. 2, pp. 280-290, 2014.

[25] J. C. Ryan, A. G. Banerjee, M. L. Cummings, and N. Roy, "Comparing the performance of expert user heuristics and an integer linear program in aircraft carrier deck operations," IEEE Transactions on Cybernetics, vol. 44, no. 6, pp. 761-773, 2014.

[26] Y. Wu and X. Qu, "Path planning for taxi of carrier aircraft launching," Science China Technological Sciences, vol. 56, no. 6, pp. 1561-1570, 2013. 


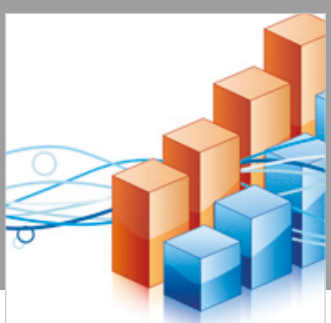

Advances in

Operations Research

\section{-n-m}
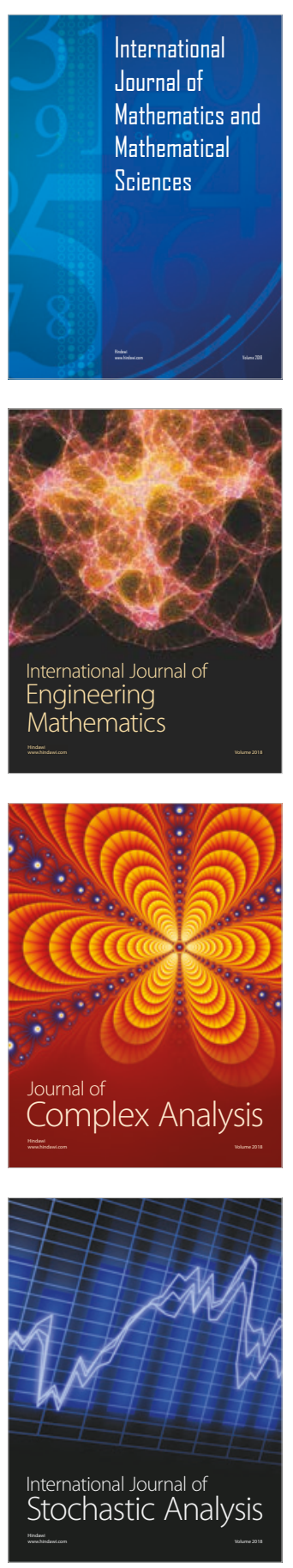
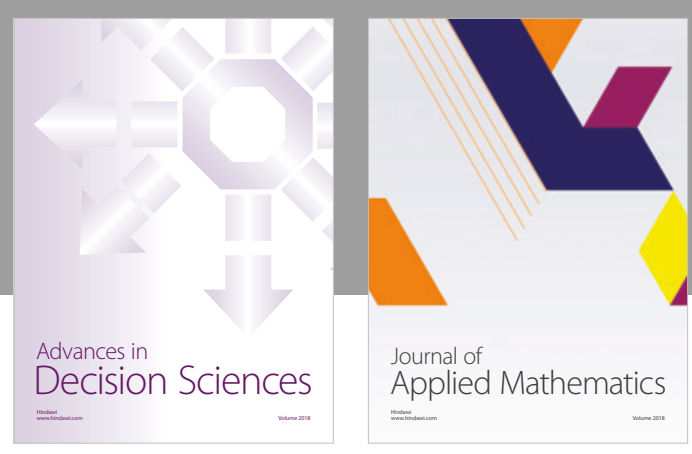

Journal of

Applied Mathematics
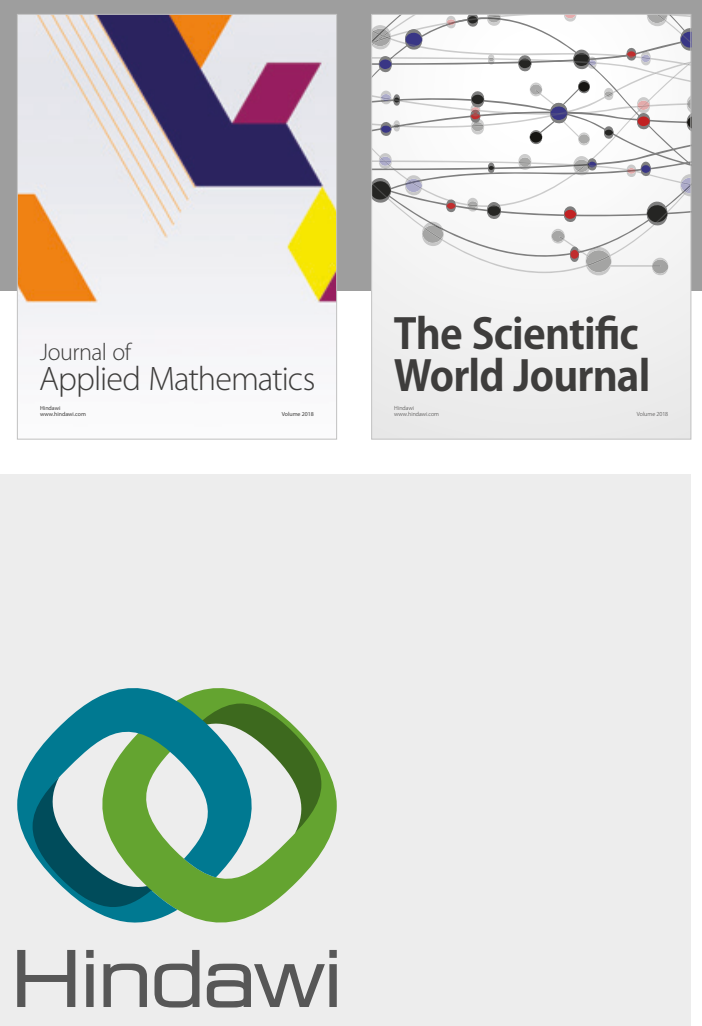

Submit your manuscripts at

www.hindawi.com

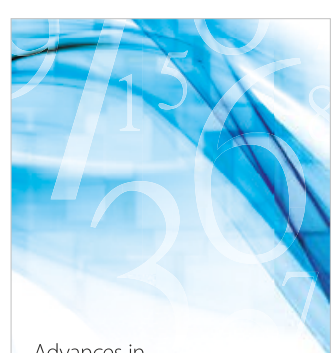

Advances in
Numerical Analysis
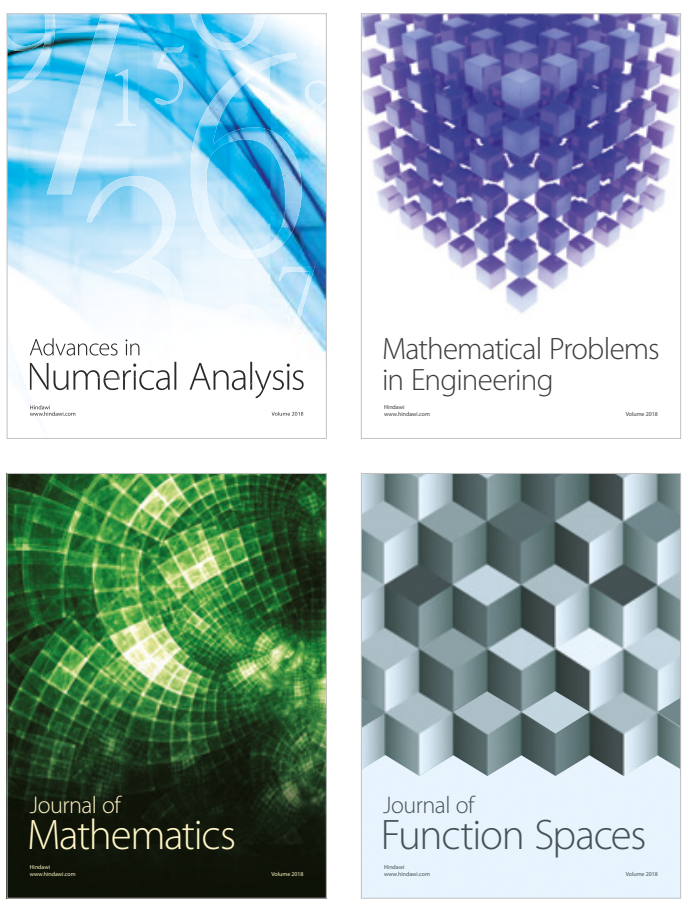

Mathematical Problems in Engineering

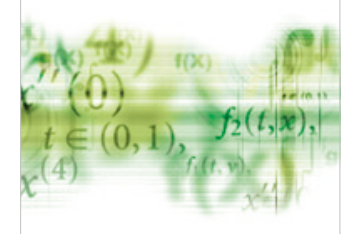

International Journal of

Differential Equations

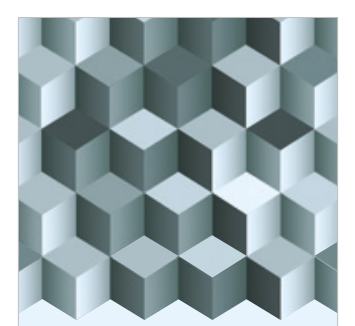

Journal of

Function Spaces

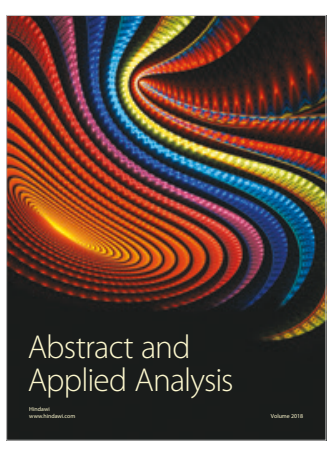

The Scientific

World Journal

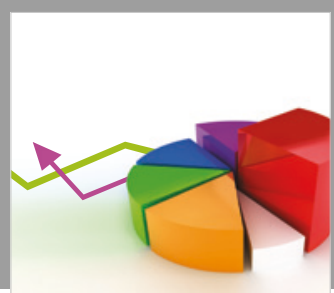

Journal of

Probability and Statistics
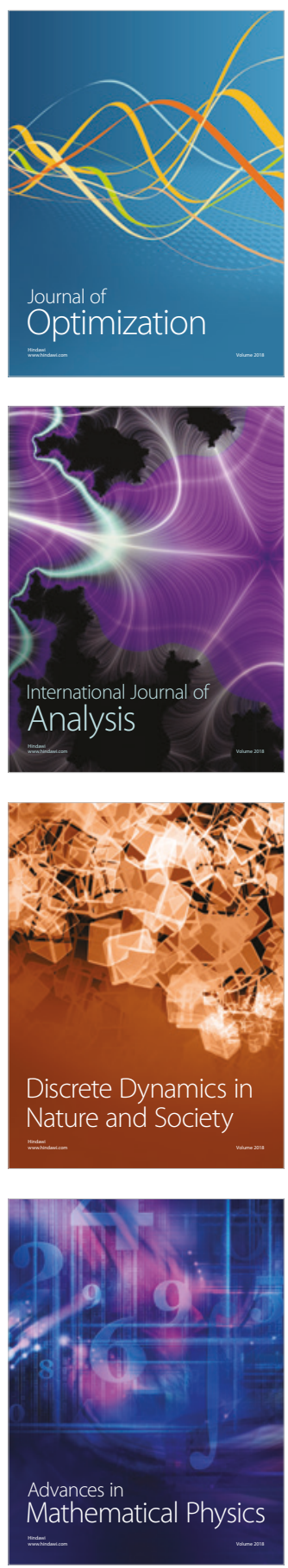